\title{
Measuring the Characteristic Length Scale of Medium Range Order in Amorphous Silicon Using Variable Resolution Fluctuation Electron Microscopy
}

\author{
L. N. Nittala*, R. D. Twesten ${ }^{* *}$, P. M. Voyles ${ }^{* * *}$ and J. R. Abelson ${ }^{*}$ \\ * Department of Materials Science and Engineering, University of Illinois, Urbana, Illinois 61801 \\ ** Center for Microanalysis of Materials, University of Illinois, Urbana, Illinois 61801 \\ *** Department of Materials Science and Engineering, University of Wisconsin, Madison, Wisconsin \\ 53706
}

Recent studies using fluctuation electron microscopy [1] have revealed the presence of medium range structural order (MRO) in amorphous silicon (a-Si) thin films. Such order is not predicted by the continuous random network model [2], and may have implications for the nature of electronic transport in hydrogenated a-Si, which is used as the semiconductor in large-area electronics [3]. The paracrystalline model ascribes this MRO to the presence of nanoscale regions that are topologically crystalline but may be strained [4]. Here we analyze the length scale of these ordered regions in a-Si using the variable resolution mode of fluctuation electron microscopy implemented on a JEOL 2010F TEM [5]. To accomplish this, we developed a set of operating conditions that can be used on a routine basis.

Fluctuation microscopy is a probe-based scattering technique that quantifies structural order on the 1-3 nm length scale in disordered materials [6]. The statistical variance $\mathrm{V}$ of the scattered intensities from many nanoscale volumes is calculated as a function of the scattering vector $\mathrm{k}$. Previous fluctuation microscopy experiments in a TEM used hollow cone dark field imaging at a fixed real space resolution to measure $\mathrm{V}(\mathrm{k})$. However, determining the paracrystallite size in a-Si requires $\mathrm{V}(\mathrm{k}, \mathrm{Q})$ data, where $\mathrm{Q}$ is the objective (or probe-forming) aperture radius in reciprocal space, and the real space resolution $\mathrm{R}=0.61 / \mathrm{Q}$. Measuring $\mathrm{V}(\mathrm{Q})$ at fixed $\mathrm{k}$ is called variable resolution fluctuation electron microscopy (VR-FEM) [5]. Qualitatively, if the probe size is exactly matched to the size of the paracrystallites, we expect a maximum in $\mathrm{V}(\mathrm{Q})$. Real materials are likely to contain a distribution of sizes, but systematic measurements as a function of probe size will still provide a characteristic scale of the size distribution.

Following Voyles and Muller [5], we have implemented VR-FEM on a Schottky emission JEOL 2010F. The JEOL 2010 has a condenser minilens capable of forming electron probes with varying convergence angles. Highly coherent diffraction-limited electron probes with a full width at half maximum in the range 1-4 $\mathrm{nm}$ were obtained using a very high demagnification of the source and a range of settings for the condenser minilens. Two different condenser apertures, $4 \mu \mathrm{m}$ and $10 \mu \mathrm{m}$, were used to define the probe sizes. The objective lens setting was fixed at the value optimized for high resolution imaging. The probes were focused on the sample using Ronchigrams and the condenser aperture was centered on the coma free axis.

Nanodiffraction patterns were recorded using a Gatan CCD camera. The acquisition of nanodiffraction patterns was automated to systematically vary the condenser deflectors and scan the sample area in steps of $5 \mathrm{~nm}$ in a square grid of 10x10 points. The exposure time was set to $\sim 6$ seconds and a total of $\sim 500$ patterns were collected in five sets of $10 \times 10$ patterns from different areas of the sample to get a statistically significant representation and a good signal to noise ratio. The nanodiffraction patterns were analyzed by azimuthally averaging each image to get the average intensity, $\mathrm{I}(\mathrm{k})$, as a function of the scattering vector. The variance in $\mathrm{I}(\mathrm{k})$ was calculated for each set 
of patterns. The final $\mathrm{V}(\mathrm{k})$ plot represents an average of the five data sets with the error bars defined by one standard deviation of the mean.

Figure 1(a) shows the $\mathrm{V}(\mathrm{k})$ data from a $\sim 20 \mathrm{~nm}$ thick a-Si thin film deposited at a substrate temperature of $230^{\circ} \mathrm{C}$ by DC magnetron sputtering. The probe sizes of $1.2,1.5,2.3$ and $3.6 \mathrm{~nm}$ represent virtual objective apertures with radii of $0.051,0.041,0.026$, and $0.017 \AA^{-1}$, respectively. The variation in the $\mathrm{V}(\mathrm{k}, \mathrm{Q})$ signal with probe size is an indication of the varying sensitivity of the probes to paracrystalline regions with a particular size. Gibson et al. have predicted that $V(Q)$ data should follow the relationship

$$
\frac{Q^{2}}{V(k, Q)}=\left(\frac{1}{\Lambda^{3} P(k)}\right)+\left(\frac{4 \pi^{2}}{\Lambda P(k)}\right) Q^{2},
$$

which implies that $\mathrm{Q}^{2} / \mathrm{V}$ graphed against $\mathrm{Q}^{2}$ should be linear, and a characteristic length $\Lambda$ can be extracted from the slope and intercept of the line [7]. This has been tested in simulation [7], but not experimentally. Figure $1(\mathrm{~b})$ is a plot of our data at $\mathrm{k}=0.31 \AA^{-1}$ in this form, and a linear fit with correlation coefficient $\mathrm{R}=0.86$. From this fit, the characteristic length scale for this sample is $\Lambda=$ $1.0 \pm 0.1 \mathrm{~nm}$. We will report additional experiments of this type on the evolution of $\Lambda$ as a function of deposition conditions or exposure to atomic hydrogen.

\section{References}

[1] P. M. Voyles and J. R. Abelson, Sol. Energy Mater. Sol. Cells 78 (2003) 85.

[2] P. M. Voyles et. al., Phys. Rev. Lett. 86 (2001) 5514.

[3] R.A. Street, Hydrogenated Amorphous Silicon, Cambridge University Press, 1991.

[4] P. M. Voyles et al., J. Appl. Phys. 90 (2001) 4437.

[5] P. M. Voyles and D. A. Muller, Ultramicroscopy 93 (2001) 147.

[6] P.M. Voyles et al., J. Electron Microsc. 49 (2000) 259.

[7] J. M. Gibson et. al., Ultramicroscopy 83 (2000) 169.

[8] This project is supported by the National Science Foundation (NSF DMR 02-05858) and was carried out in the Center for Microanalysis of Materials, University of Illinois, which is partially supported by the U.S. Department of Energy (DEFG02-91- ER45439). We thank S. Jayaraman for supplying the a-Si thin films.
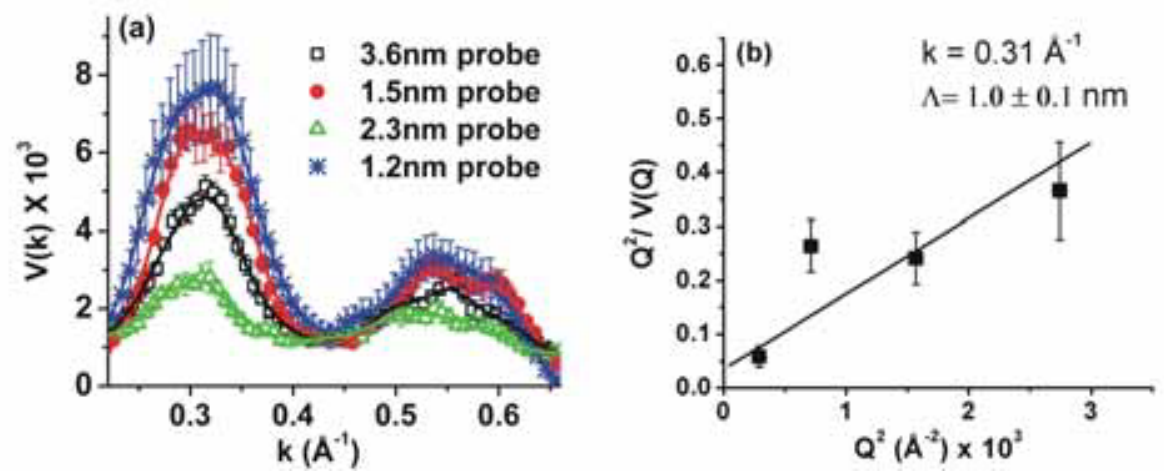

Figure 1: (a) VR-FEM measurements on an a-Si thin film using four different probes sizes. The real space resolution of the data is equivalent to the probe size. (b) The VR-FEM data at $\mathrm{k}=0.31 \AA^{-1}$ plotted along with the resolution function $Q$. The slope and intercept of the linear fit are used to estimate the characteristic length scale $\Lambda$. 